\title{
Renal involvement in granulomatosis with polyangiitis
}

\begin{abstract}
Granulomatosis with polyangiitis (GPA) is defined by the presence of small vessel vasculitis that affects the upper respiratory tract, lungs and kidneys. Renal involvement is frequent. The disease varies from asymptomatic to fulminant rapidly progressive glomerulonephritis forms, leading to terminal renal failure in days.
\end{abstract}

Volume 8 Issue 6 - 2020

\author{
Aya Fraj, Olfa Berriche, Sondes Arfa, Jihen \\ Chelli,Wafa Bel Abed, MH Sfar \\ Taher Sfar Hospital,Tunisia
}

Correspondence: Olfa Berriche, Taher Sfar Hospital, Tunisia, Email olfaberrihe@gmail.com

Received: December 07, 2020 | Published: December 2I, 2020

\section{Introduction}

Granulomatosis with polyangiitis (GPA) is described by the Chapel Hill nomenclature as a systemic necrotizing vasculitis affecting small sized vessels. It is characterized by the frequency of the upper respiratory tract, lungs and the kidneys involvement. The prevalence of renal manifestations is variable, ranging from 45 to $90 \%{ }^{1}$ usually within the first two years of the disease onset. ${ }^{2}$ It depends on the origin of patient recruitment, the classification criteria used to label renal disease, and the duration of follow-up. The aim of this study is to determine the clinical course of patients with GPA and renal involvement.

\section{Methods}

We performed a retrospective study of 11 patients followed for GPA in the Internal Medicine Department of Taher Sfar Hospital Mahdia and Fattouma Bourgiba Hospital Monastir, during a period of 10 years. All patients fulfilled the ACR classification criteria. ${ }^{3}$

\section{Results}

There were 6 men and 5 women with a mean age of 47 years. Clinical features were various. Ear,nose,throat (ENT) signs were present in $100 \%$ of cases and, general signs in $90,9 \%$ of cases, lung manifestations were described in $72,7 \%$ of cases, joint involvement was reported in $63,3 \%$ of cases, eye involvement was observed in $63,6 \%$ of cases , kidney involvement was seen in $45,4 \%$ of cases and cutaneous involvement was present in $45.4 \%$ of cases. Renal involvement was observed in 5 patients $(45.4 \%)$. It was discovered at the onset of the disease in all patients. It variously associated renal failure in 4 cases $(36.3 \%)$, microscopic hematuria in 5 cases $(45.4 \%)$ and proteinuria in 3 cases $(27.2 \%)$.

Renal biopsy was performed in 5 cases. The characteristic finding in patients with GPA was extra capillary glomerulonephritis with exudative deposition of neutrophils and a crescent in two cases, segmental and focal hyalinosis in one case, vasculitis with hemorrhagic suffusions associated with a granulomatous reaction without necrosis was observed in one patient and it was inconclusive in the remaining case. The c-ANCA were positive in all cases $(5 / 5)$. During the induction phase, all patients received corticosteroids and cyclophosphamide. Cotrimoxazole was also given to all these patients immediately. In the maintenance phase, the only drug used was azathioprine in all cases. Four patients $(80 \%)$ achieved remission, while relapses were seen in one case $(20 \%)$. The median time to remission was 3 months. One death was reported to be due to severe sepsis and kidney impairment.

\section{Discussion}

Granulomatosis with polyangiitis (GPA) is a small vessel necrotizing vasculitis, which involving both the upper and lower respiratory tracts and the kidneys. The pathogenesis of GPA is still unknown, although an autoimmune response appears to be involved in the development of the disease. The kidney is the most commonly affected vital organ in GPA, and patient outcomes are largely determined by the severity of renal disease at diagnosis and by its response to treatment. ${ }^{4}$ Rapidly progressive glomerulonephritis is the most frequent clinical manifestation of GPA, although the syndromes of asymptomatic hematuria with proteinuria, acute kidney injury, chronic kidney disease or end-stage renal failure or acute nephritis are possible as well. ${ }^{5}$

The most characteristic histological feature of GPA is the presence of necrotizing granulomatous inflammation, but this finding is usually not found in the kidney. ${ }^{6}$ Renal disease in GPA is almost always a pauci-immune necrotizing and crescentic glomerulonephritis. The lesions are focal or diffuse, with scanty or absent glomerular staining for immunoglobulin by immunofluorescence microscopy. ${ }^{7}$ Left untreated, GPA is a fatal condition, as renal failure secondary to renal involvement reduces the prognosis for recovery. Therapy for GPA has increased survival, resulting in remission in more than $90 \%$ of patients. ${ }^{8}$ If untreated, GPA usually runs a rapidly fatal course, and $82 \%$ of patients die within one year. Chronic renal insufficiency developed in $42 \%$ of patients, and $11 \%$ of patients required dialysis, while $5 \%$ had to undergo renal transplantation. ${ }^{7}$ Thus, accurate and early diagnosis is of primordial importance to ameliorate the prognosis of this disease to effective therapy, and effective therapy leads to longer patient survival. ${ }^{5}$

\section{Conclusion}

Recently, a good progress has been made in understanding the epidemiology, environmental and genetic risk factors of the role of antineutrophil cytoplasmic antibodies (ANCA) in kidney 
pathogenesis and the utilization of ANCA in diagnosis. The kidney is the most commonly affected vital organ in GPA, and patient outcomes are largely determined by the severity of renal disease at diagnosis and by its response to treatment.

\section{Acknowledgments}

None.

\section{Conflicts of interest}

The author declares there is no conflict of interest.

\section{References}

1. Karras A, Guiard E, Lévi C, et al. Granulomatose avec polyangéite (maladie de Wegener). Presse Médicale. 2012;41(10):1014-1023.

2. Hoffman GS, G S Kerr, R Y Leavitt, et al. Wegener Granulomatosis: An Analysis of 158 Patients. Ann Intern Med. 1992;116(6):488-498.
3. Leavitt RY, Fauci AS, Bloch DA, et al. The American College of Rheumatology 1990 criteria for the classification of wegener's granulomatosis. Arthritis Rheum. 2010;33(8):1101-1107.

4. Shoichi Ozaki. ANCA-associated Vasculitis: Diagnostic Therapeutic Strategy. Allergology International. 2007;56(2):87-96.

5. Greco A, Marinelli C, Fusconi M, et al. Clinic manifestations in granulomatosis with polyangiitis. Int $J$ Immunopathol Pharmacol. 2016;29(2):151-159.

6. Charles Jennette J, J Falk R. The Pathology of Vasculitis Involving the Kidney. Am J Kidney Dis. 1994;24(1):130-141.

7. Samarkos M, Loizou S, Vaiopoulos G, et al. The Clinical Spectrum of Primary Renal Vasculitis. Semin Arthritis Rheum. 2005;35(2):95-111.

8. Andrassy K, Erb A, Koderisch J, et al. Wegener's granulomatosis with renal involvement: patient survival and correlations between initial renal function, renal histology, therapy and renal outcome. Clin Nephrol. 1991;35(4):139-147. 